09

\title{
Бинарные фазовые транспаранты на основе жидкокристаллической матрицы видеопроектора
}

\author{
(C) М.С. Кузьмин, С.А. Рогов व \\ Санкт-Петербургский политехнический университет Петра Великого, \\ 195251 Санкт-Петербург, Россия \\ e-mail: sarogov@mail.ru
}

(Поступило в Редакцию 27 марта 2017 г.)

\begin{abstract}
Продемонстрирована возможность бинарной фазовой модуляции в жидкокристаллических матрицах, серийно выпускаемых для видеоаппаратуры. Экспериментально получены и исследованы элементы плоской дифракционной оптики - цилиндрические и сферические линзы. Рассмотрены ограничения, связанные с конечным размером пикселя бинарного дифракционного транспаранта.
\end{abstract}

DOI: $10.21883 /$ JTF.2018.01.45487.2268

Плоские фазовые транспаранты могут использоваться в когерентных оптических системах в виде дифракционных оптических элементов (дифракционных линзах и фокусаторах), в компенсаторах фазовых искажений, в качестве фазовых пространственных модуляторов света и чисто фазовых фильтров для систем обработки информации [1-4].

Транспаранты, управляемые электрическим напряжением, некогерентным светом или сканирующим лазерным лучом, могут изменять функцию пропускания за достаточно короткое время. Одними из наиболее перспективных перестраиваемых устройств являются жидкокристаллические (ЖК) матрицы с электронным управлением. Они имеют малые габариты и вес, небольшие управляющие напряжения, достаточно большое число разрешимых точек. Выпуск таких матриц и устройств управления к ним освоен промышленностью для видеоаппаратуры. Эти матрицы существенно дешевле специальных ЖК устройств, разработанных для когерентной оптики [5]. Эксперименты показали, что оптическое качество матриц, выпускаемых для видеоаппаратуры, часто удовлетворяет требованиям работы в когерентных системах [6], кроме того, при использовании фазовой модуляции в матрице возможна попутная компенсация ее фазовых неоднородностей соответствующей коррекцией управляющих напряжений на пикселях.

Для амплитудной модуляции в ЖК матрицах используется вращение плоскости поляризации проходящего света, амплитуда света на выходе зависит от величины управляющего напряжения и положений входного и выходного поляризаторов [7]. Наряду с амплитудной в ЖК матрице происходит также фазовая модуляция света, величина которой связана с двулучепреломлением в жидком кристалле. Выбирая положение поляризаторов и диапазон изменения напряжения, прилагаемого к кристаллу, можно добиться преимущественно фазовой модуляции [8]. Максимально возможная глубина фазовой модуляции для многих типов матриц не достигает величины $2 \pi$, необходимой для создания транспарантов с произвольным фазовым распределением. Поворот фазы можно увеличить, если организовать многократное прохождение света через матрицу [9], однако при использовании внешних отражающих зеркал такая система сложна и труднореализуема.

Достаточно просто организовать в ЖК матрице бинарную фазовую модуляцию. Если положение оси поляроида на выходе матрицы выбрать перпендикулярным биссектрисе угла между направлениями векторов поляризации проходящего света при минимальном и максимальном напряжениях, то проекции этих векторов на ось поляроида будут направлены навстречу друг другу (рис. 1), а фаза света, проходящего через пиксели с минимальным и максимальным управляющими напряжениями будет отличаться на $180^{\circ}$ [10]. Последнее условие выполняется, если пренебречь фазовой задержкой

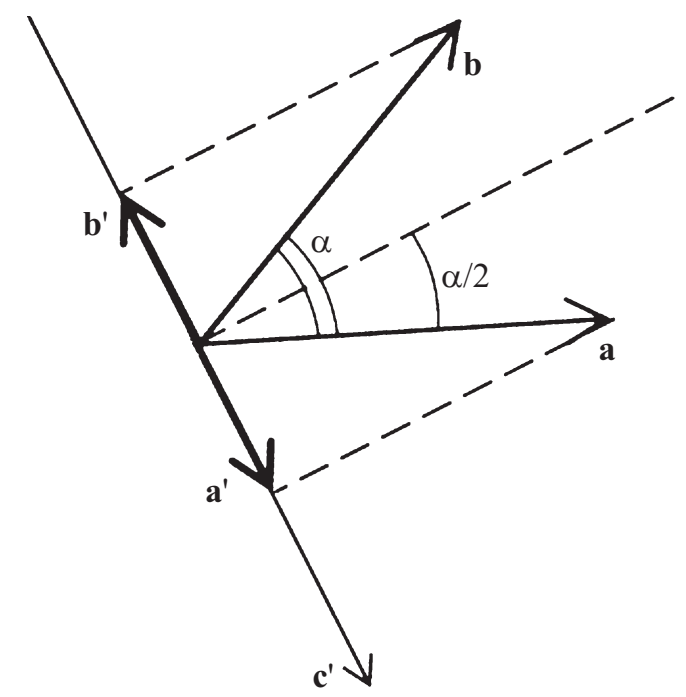

Рис. 1. Векторы поляризации света на выходе жидкокристаллической матрицы (a - для максимального управляющего напряжения, $\mathbf{b}$ - для минимального управляющего напряжения) и их проекции на выходной поляризатор при бинарной фазовой модуляции. Направление осей входного и выходного поляризаторов совпадает с а и с. 

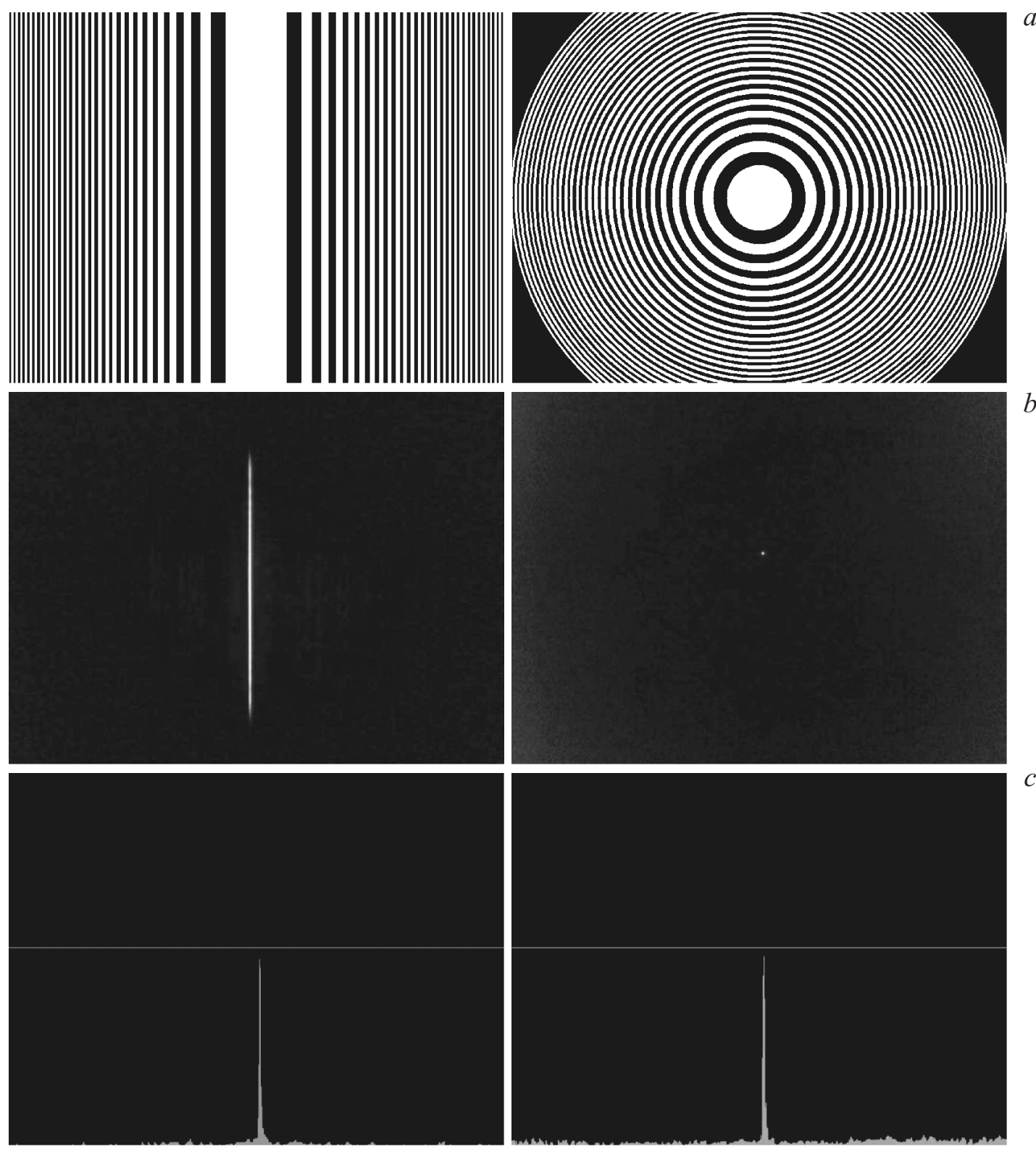

Рис. 2. $a-$ амплитудный аналог бинарных фазовых зонных пластинок, сформированных в ЖК матрице, $b-$ распределения света в фокусах первого порядка, $c$ - распределения интенсивности в сечениях этих распределений.

между рассматриваемыми проекциями, возникающей изза двойного лучепреломления. Эксперименты показали, что подбором положения входного и выходного поляризаторов вблизи рассматриваемой геометрии можно добиться противоположной фазы проходящего света при двух значениях управляющего напряжения.

Нами проверялась возможность получения управляемой бинарной фазовой модуляции в ЖК матрице видеопроектора. Число элементов данной матрицы составляло $1024 \times 768$, скорость переключения матрицы - 70 кадров в секунду. Максимальный угол поворота вектора поляризации света в диапазоне управляющих напряжений составил $45^{\circ}$. Оптимальные для бинарной фазовой модуляции углы осей входного и выходного поляризаторов оказались равными 90 и $160^{\circ}$ (геометрия, близкая к показанной на рис. 1).

Бинарные фазовые элементы (с фазовой задержкой 0 и $\pi \mathrm{rad})$ требуют меньшей разрешающей способности от транспаранта, чем элементы плоской оптики с более точной передачей функции фазовой задержки [1]. Примером таких фазовых транспарантов являются зонные пластинки Френеля, которые могут использоваться в качестве линз. Платой за уменьшение разрешающей способности являются дополнительные фокусы линз, которые присущи этим дифракционным элементам и которые создают мешающий фон в плоскости фокусировки линзы. Чисто фазовые бинарные дифракционные транспаранты имеют преимущества по отношению сигнал- 
шум перед предложенными в работе [11] аналогами с амплитудным кодированием, так как у них отсутствует нулевой порядок и их дифракционная эффективность выше.

На рис. 2, $a$ показаны амплитудные аналоги сформированных в ЖК матрице фазовых зонных пластинок, соответствующих цилиндрической и сферической линзам. Темные и светлые зоны соответствовали минимальному и максимальному уровням серого на пикселях. На рис. $2, b$ приведены распределения света в фокусах первого порядка этих дифракционных линз, а на рис. 2, $c-$ распределения интенсивности в сечениях фокальных распределений. Отношение сигнал-шум в фокусе бинарной цилиндрической линзы - цилиндрической линзы составило 27, а для бинарной линзы с амплитудным кодированием - 14 .

Особенностью голограмм, формируемых с помощью ЖК матриц с электронным управлением, является сравнительно низкая разрешающая способность, связанная с конечным размером пикселя $(\sim 10 \mu \mathrm{m})$. Этот размер определяет минимальный период зон (штрихов) дифракционной решетки, который может быть получен: два пикселя на период. В голограммах, к которым можно отнести и дифракционные линзы, имеет значение не только период штрихов, но и их положение, определяющее фазу дифрагированного света. Допустимой фазовой ошибкой в оптических элементах считается величина $(\pi / 7-\pi / 2) \mathrm{rad}[12]$. Будем считать, что предельно допустимой фазовой ошибкой является $\pi / 2 \mathrm{rad}$, что соответствует сдвигу штрихов дифракционной решетки на четверть периода. Такой сдвиг штриха возникает при возможном в процессе записи решетки ошибочном отнесении пикселя к соседней зоне, если на одну зону приходится два пикселя ЖК матрицы, т. е. четыре пикселя на период.

В дифракционных линзах размер пикселя накладывает ограничение на предельную апертуру линзы, поскольку частота штрихов плоской линзы увеличивается при удалении от ее центра. Приравнивая период зонной структуры на краю плоской линзы максимального размера к четырем размерам пикселя, получим

$$
\frac{x_{\max }}{f} \leq \frac{\lambda}{4 \Delta}
$$

где $x_{\max }-$ половина максимальной апертуры линзы, $f$ - фокусное расстояние линзы, $\lambda$ - длина волны света, $\Delta$ - размер пикселя. Выражение (1) дает максимальное значение относительного отверстия дифракционной линзы при данном размере пикселя.

Для экспериментальной проверки выражения (1) бинарные дифракционные цилиндрические линзы с разным фокусным расстоянием формировались на ЖК матрице под управлением компьютера [13], размер матрицы $(10.5 \mathrm{~mm})$ превосходил значение $2 x_{\max }$. Матрица освещалась плоской волной когерентного света через щель размером $2 x_{\max }$ ехр, и в фокусе сформированной линзы измерялась ширина распределения света. Ширина $2 x_{\max }$ exp
Предельные теоретические и экспериментальные значения максимальной апертуры дифракционной цилиндрической линзы с допустимыми аберрациями для разных фокусных расстояний

\begin{tabular}{l|c|c|c}
\hline$f$ & 150 & 232 & 370 \\
\hline $2 x_{\max }$ & 4.8 & 7.6 & 12.0 \\
\hline $2 x_{\max \exp }$ & 5.8 & 6.6 & 10.4
\end{tabular}

увеличивалась до тех пор, пока ширина фокального пятна не переставала уменьшаться. Экспериментальные значения $x_{\max } \exp$ хорошо совпадали с полученными из формулы (1) значениями $x_{\max }$ (таблица, все величины в таблице даны в $\mathrm{mm})$.

Аналогичные ограничения, связанные с конечной шириной пикселей, возникают и при других применениях бинарных фазовых транспарантов. Так, при вводе в оптическую систему пространственного сигнала с помощью пространственного модулятора света, ширина пикселей модулятора будет ограничивать максимальную пространственную частоту в спектре вводимого сигнала. При этом в случае, когда важна и фаза частотных компонентов (например, в схеме пространственной фильтрации), ширина пикселя должна быть не более четверти пространственного периода максимальной частоты спектра входного сигнала.

При создании бинарных чисто фазовых фильтров ширина пикселей будет ограничивать максимальные пространственные частоты в фильтрах, соответствующие деталям импульсного отклика, наиболее удаленным от его центра, т.е. размеры импульсного отклика. В случае необходимости сохранения фазовых соотношений в импульсном отклике, также могут быть получены требования к ширине пикселя, аналогичные рассмотренным выше.

Таким образом, проведенное исследование показало возможность создания бинарных чисто фазовых транспарантов на основе ЖК матриц для видеоаппаратуры с учетом рассмотренных ограничений, связанных с конечной разрешающей способностью этих матриц.

\section{Список литературы}

[1] Казанский Н.Л. Автореф. канд. дис. Анализ характеристик дифракционных оптических элементов, фокусирующих лазерное излучение. 01.04.01. 1996. 229 с.

[2] Кольер Р., Беркхард К., Лин Л. Оптическая голография. М: Мир, 1973. 686 c.

[3] Gouwen Lu, Yu F.T.S. // Appl. Opt. 1996. Vol. 35. N 2.

[4] Фленнери Д.Л. // ТИИЭР. 1989. Т. 77. № 10. С. 136.

[5] Holoiye. Preliminary specification sheet XGA Spatial Light Modulator Kit LC-R 2012 // HOLOEYE Photonics AG.

[6] Kuzmin M.S., Rogov S.A. // Optical Memory \& Neural Networks (Information Optics). 2013. Vol. 22. N 4. P. 261266. 
[7] Электронный ресурс. Жидкокристаллический дисплей. Режим доступа: ru.wikipedia.org. Википедия - свободная энциклопедия.

[8] Lu K., Saleh B.E.A. // Opt. Eng. 1990. Vol. 29. P. 240-246.

[9] Barnes T.H., Eiju T., Matsuda K., Ooyama N. // Appl. Opt. 1989. Vol. 28. P. 4845-4852.

[10] Davis J.A., Heissenberger G.M., Lilly R.A., Cottrell D.M., Brownell M.F. // Apl. Opt. 1987. Vol. 26. P. 929-933.

[11] Flavin M.A., Horner J.L. // Appl. Opt. 1989. Vol. 28. N 9. P. 1692-1696.

[12] Борн М., Вольф Э. Основы оптики. М.: Наука, 1970. 856 с.

[13] Кузьмин М.С., Рогов С.А. // Опт. журн. 2015. Т. 82. № 3. C. 23-29. 